\title{
COMPARACIÓN DE ESTIMADORES NO PARAMÉTRICOS FRENTE A LOS PARAMÉTRICOS PARA LA FUNCIÓN DE CONFIABILIDAD
}

\section{(Comparison of nonparametric estimators versus parametric for reliability function)}

\author{
Javier Ramírez Montoya*, Guillermo Martínez Flórez**
}

*Departamento de Matemáticas y Física. Grupo Investigación en Estadística y Aplicaciones. GINESAP, javier.ramirez@unisucre.edu.co. **Departamento de Matemáticas y Estadística, Grupo Investigación en Estadística, gmartinezflorezgm@correo.unicordoba.edu.co

(Recibido febrero 13 de 2015 y aceptado abril 6 de 2015)

\begin{abstract}
Resumen:
Uno de los principales objetivos del área de confiabilidad es estimar la función de confiabilidad, donde tradicionalmente se utilizan estimadores no paramétricos, que son más eficientes en tamaños de muestras considerables. En este trabajo se comparan los estimadores no paramétricos para la función de confiabilidad a través del error cuadrático medio, utilizando los estimadores no paramétricos de Kaplan y Meier (1958), el estimador de Nelson (1969) y Bootstrap aplicado a Kaplan y Meier y Nelson. La comparación se hace teniendo en cuenta las estimaciones paramétricas, mediante simulación con diferentes escenarios, tiempos de interés, tamaños de muestra y porcentajes de censura, y muestra que el remuestreo Bootstrap tipo normal no presenta los mejores resultados con Kaplan y Meier (1958). Y mediante Nelson (1969), el $18 \%$ fue más eficiente.
\end{abstract}

Palabras clave: Bootstrap, confiabilidad, estimadores no paramétricos.

\section{INTRODUCCIÓN}

En el análisis de confiabilidad tradicional, también llamado análisis de supervivencia en el área de las ciencias médicas, con frecuencia resulta muy informal la utilización de estimadores no paramétricos para estimar la función de confiabilidad cuando no se tiene una distribución aproximada para la variable en estudio

\section{Abstract:}

One of the main objectives of the area of realiability is to estimate the function of reliability, where traditionally are used non parametric estimators, being more efficient in sample of big sizes. In this work nonparametric estimators are compared to the reliability function through the mean square error using nonparametric estimators of Kaplan \& Meier (1958), Nelson estimator (1969) and Bootstrap applied to Kaplan \& Meier and Nelson. The comparison is made considering the parametric estimates, through simulation with different scenarios, times of interest, sizes sample and percentages of censorship, showing that the Bootstrap resampling normal type does not present the best results with Kaplan \& Meier. Using Nelson, the $18 \%$ was more efficient.

Keywords: Bootstrap, reliability, nonparametric estimators.

y más aún sin pruebas de diagnóstico de la variable, si se tiene en cuenta que su escogencia depende de la estructura de la variable estudiada. Por lo tanto, conocer las condiciones o escenarios donde los estimadores no paramétricos presentan mejores resultados, es muy importante en la práctica, puesto que permitiría establecer la sensibilidad de la estimación en situaciones extremas, es decir, cuando son más permisibles los procedimientos 
máximos verosímiles con observaciones censuradas frente a los de principio de independencia.

Arrabal, Dos Santos, Da Rocha, Nonaka y Meira (2014) revisan los métodos de remuestreo comparándolos mediante intervalos de confianza, que en el trabajo más recientemente encontrado, enfatizan en la regresión de Cox, con datos censurados. Sin embargo, en este trabajo hacen la comparación mediante la medida de eficiencia del error cuadrático medio, tomando como referencia la estimación paramétrica del modelo generador, y teniendo en cuenta más factores.

Por otra parte, He, Kong y Su (2013), con base en datos censurados a derecha, estiman la función de confiabilidad a trozos, según distintos tamaños muestrales, y determinan la gravedad en la estimación con datos censurados, en el caso exponencial. Tener información sobre los comportamientos de los estimadores no paramétricos para la función de confiabilidad en diferentes escenarios, en particular para las áreas de ingeniería y ciencias médicas, es de vital importancia, puesto que las decisiones basadas en malas estimaciones podrían traer catástrofes.

Este experimento es desarrollado vía simulación, debido a las diferentes funcionalidades de los estimadores no paramétricos, además de las expresiones que se obtienen; por lo tanto, es la estrategia más adecuada.

\section{FUNCIÓN DE CONFIABILIDAD}

La función de confiabilidad o también llamada función de sobrevivencia en el campo de las ciencias médicas, permite obtener la probabilidad de ocurrencia de evento después del tiempo de interés $t_{0}$. En confiabilidad pretende cuantificar el funcionamiento de un sistema bajo condiciones de operación. Sea $T$ una variable aleatoria que representa el tiempo de ocurrencia de un evento cualquiera, luego para un tiempo de interés $t_{0}$, se define como $P\left(T>t_{0}\right)$.

\section{ESTIMADORES NO PARAMÉTRICOS}

\subsection{Estimador de Kaplan-Meier}

Kaplan y Meier (1958) proponen una modificación de $\hat{S}(t)$ a la cual denominaron estimador del producto límite (EPL) de la función de confiabilidad. En efecto, supóngase que existen observaciones de $\mathrm{n}$ individuos y que hay $\mathrm{k} \leq \mathrm{n}$ tiempos distintos en los cuales la muerte ocurre, esto es, $\mathrm{t} 1<t 2<\cdots<t k$.

Se admite la posibilidad de tener más de una muerte en $t_{i}$ y $d_{j}$ representará el número de muertes en $t_{j}$. Además, existen los tiempos de censura $t_{c}$ para individuos cuyo tiempo de vida no es observado (Colosimo \& Giolo, 2006).

El estimador del producto límite $\hat{S}(t)$, es definido como:

$$
\hat{S}(t)=\prod_{j: t_{j}<t}^{k} \frac{n_{j}-d_{j}}{n_{j}}
$$

donde $n_{j}$ es el número de individuos en riesgo en $t_{j}$, es decir, el número de individuos vivos y no censurados justo antes de $t_{j}$. Cualquier individuo con tiempo de censura registrado igual a $t_{j}$ será incluido en el conjunto de $n_{j}$ individuos en riesgo en $t_{j}$, como individuos que murieron en $t_{j}$. Esta convención es razonable, puesto que un individuo censurado en el tiempo $t_{c}$ casi ciertamente sobrevive después de $t_{c}$, (Lawless, 2003).

3.1.1 Varianza estimador Kaplan-Meier. Para evaluar los resultados eficazmente cuando se usa el estimador del producto límite, es conveniente tener un estimador de la varianza de $\hat{S}(t)$. Una de las utilizadas en este trabajo es la fórmula de Greenwood (1926), dada por:

$$
\widehat{\operatorname{Var}}(\hat{S}(t))=\hat{S}^{2}(t) \sum_{j: t_{j}<j}^{k} \frac{d_{j}}{n_{j}\left[n_{j}-d_{j}\right]}
$$

La derivación de (2) se realiza mediante el método delta:

$$
\begin{aligned}
\log (\hat{S}(t))=\log \left(\prod_{j: t_{j}<t}^{k} \frac{n_{j}-d_{j}}{n_{j}}\right) \\
=\sum_{j=1}^{k} \log \left(\frac{n_{j}-d_{j}}{n_{j}}\right) \\
=\sum_{j=1}^{k} \log \left(p_{j}\right)
\end{aligned}
$$


Entonces,

$$
\begin{array}{r}
\operatorname{Var}(\log (\hat{S}(t))) \\
=\sum_{j=1}^{k} \operatorname{Var}\left(\log \left(p_{j}\right)\right)
\end{array}
$$

Aplicando el método delta

$$
\begin{aligned}
\operatorname{Var}\left(\log \left(p_{j}\right)\right) & \approx\left(\frac{1}{p_{j}}\right)^{2} \frac{p_{j}\left(1-p_{j}\right)}{n_{j}} \\
& =\left(\frac{1}{p_{j}}\right) \frac{1-p_{j}}{n_{j}}
\end{aligned}
$$

Entonces

$$
\operatorname{Var}\left(\log \left(p_{j}\right)\right) \approx \sum_{j=1}^{k}\left(\frac{1}{p_{j}}\right) \frac{1-p_{j}}{n_{j}}
$$

Ahora

$$
\begin{aligned}
& \operatorname{Var}(\hat{S}(t)) \approx[S(t)]^{2} \operatorname{Var}(\log (\hat{S}(t))) \\
& =[S(t)]^{2} \sum_{j=1}^{k}\left(\frac{1}{p_{j}}\right) \frac{1-p_{j}}{n_{j}}
\end{aligned}
$$

Luego reemplazando $p_{j}=\frac{n_{j}-d_{j}}{n_{j}}$ se obtiene:

$\widehat{\operatorname{Var}}(\hat{S}(t))=\hat{S}^{2}(t) \sum_{j: t_{j}<j}^{k} \frac{d_{j}}{n_{j}\left[n_{j}-d_{j}\right]}$

Kalbfleisch y Prentice (1980) recomiendan realizar la estimación mediante intervalos de confianza utilizando la transformación $\boldsymbol{l o g}$.

3.1.2 Error cuadrático medio Kaplan-Meier. Con el objetivo de establecer la eficiencia del estimador no paramétrico de Kaplan-Meier, se utiliza como medida de calidad de la estimación, el error cuadrático medio, dado por:

$$
\begin{aligned}
\operatorname{ECM}\left(\hat{S}\left(t_{i}\right)\right)= & E\left[\hat{S}\left(t_{i}\right)-S\left(t_{i}\right)\right]^{2} \\
& =\left[\hat{S}\left(t_{i}\right)-S\left(t_{i}\right)\right]^{2} \\
& +\operatorname{Var}\left[\hat{S}\left(t_{i}\right)\right]
\end{aligned}
$$

donde $\hat{S}\left(t_{i}\right)$ corresponde a la ecuación (1) y $\operatorname{Var}\left[\hat{S}\left(t_{i}\right)\right]$ dado en (2) y los tiempos de interés del estudio $t_{i}=(0.2,0.4$ y 0.6$)$. Esta medida de calidad puede considerarse como la más general, ya que refleja el promedio del cuadrado de las discrepancias entre la estimación y el parámetro. El error cuadrático medio resulta la medida más recomendable en este caso, teniendo en cuenta la asimetría de las distribuciones de los tiempos de falla, reflejado en sus sesgos y cobertura de los estimadores.

\subsection{Estimador Nelson-Aalen}

Nelson (1969) inicialmente sugiere estimar la función hazard acumulada como

$$
\widehat{H}(t)=\sum_{j: t_{j}<t}^{k} \frac{d_{j}}{n_{j}}
$$

Este estimador es muy utilizado en los casos cuando se tienen tamaños de muestra pequeños

$$
\hat{S}(t)=\exp (-\widehat{H}(t))
$$

Donde, Ramírez (2011) recomienda utilizarlo cuando el tamaño de muestra es pequeño, con porcentajes de censura bajos.

3.2.1 Varianza estimador Nelson-Aalen. Una forma de calcular la varianza en la función de confiabilidad, basándose en la función hazard acumulada, es

$$
\widehat{\operatorname{Var}}(\widehat{H}(t))=\sum_{j: t_{j}<t}^{k} \frac{d_{j}\left(n_{j}-d_{j}\right)}{n_{j}{ }^{3}}
$$

Otra forma alternativa para varianza es 


$$
\widehat{\operatorname{Var}}(\widehat{H}(t))=\sum_{j: t_{j}<t}^{k} \frac{d_{j}}{n_{j}^{2}}
$$

Además, Aalen y Johansen (1978) proponen una alternativa para la estimación de la varianza de $\hat{S}(t)$, como

$$
\widehat{\operatorname{Var}}(\hat{S}(t))=\hat{S}^{2}(t) \sum_{j: t_{j}<t}^{k} \frac{d_{j}}{n_{j}{ }^{2}}
$$

En efecto,

$$
\begin{aligned}
\operatorname{Var}(\log (S(t))) & =\operatorname{Var}(-H(t)) \\
= & \operatorname{Var}\left(-\sum_{j: t_{j}<t}^{k} \frac{d_{j}}{n_{j}}\right) \\
& =\sum_{j: t_{j}<t}^{k} \frac{p_{j}}{n_{j}}
\end{aligned}
$$

$\begin{aligned} & \text { Donde } \\ & \text { orden. }\end{aligned} p_{j}=\frac{d_{j}}{n_{j}}$ aplicando método delta de segundo

$$
\begin{aligned}
& \operatorname{Var}(\hat{S}(t)) \\
& \approx[S(t)]^{2} \operatorname{Var}(\log (S(t))) \\
& =[S(t)]^{2} \sum_{j: t_{j}<t}^{k} \frac{d_{j}}{n_{j}{ }^{2}}
\end{aligned}
$$

La anterior varianza es la utilizada en este estudio.

3.2.2 Error cuadrático medio estimador de Nelson Aalen. Para el caso del estimador no paramétrico propuesto por Nelson (1969) y utilizando como medida de calidad de las estimaciones el estimador de la varianza tradicional y la alternativa de Lawless (2003) determinadas por:

$$
\begin{aligned}
\operatorname{ECM}\left(\hat{S}\left(t_{i}\right)\right)= & E\left[\hat{S}\left(t_{i}\right)-S\left(t_{i}\right)\right]^{2} \\
& =\left[\hat{S}\left(t_{i}\right)-S\left(t_{i}\right)\right]^{2} \\
& +\operatorname{Var}\left[\hat{S}\left(t_{i}\right)\right]
\end{aligned}
$$

Cuando se tienen en cuenta para la simulación diferentes factores, como en este caso, se pueden encontrar escenarios donde los estimadores tengan menor varianza pero con poca cobertura, como también mayor varianza y mayor cobertura, es indispensable tener medidas más consistentes.

\subsection{Método de remuestreo Bootstrap aplicado a datos de confiabilidad}

Un problema involucra determinar los límites de confianza para la función de confiabilidad teórica o parámetros que describen esta función. Akritas (1986) propone utilizar el método Bootstrap para estimar la confiabilidad utilizando el estimador de Kaplan y Meier (1958).

Para estimar la función de confiabilidad utilizando remuestreo al estimador de Kaplan-Meier, mediante el remuestreo Bootstrap, el proceso es el siguiente:

- Dada la muestra de tamaño $n$, estimar $\hat{S}\left(t_{i}\right)$ mediante (1). La distribución de esta muestra se considera equivalente a la distribución de la población y $\hat{S}\left(t_{i}\right)$ es el estimador muestral del parámetro poblacional $S\left(t_{i}\right)$.

- Generar $\boldsymbol{B}$ muestras Bootstrap de tamaño $n$ mediante muestreo con reemplazamiento de la muestra original, asignando a cada tiempo una probabilidad $1 / n$ y calcular los correspondientes valores $\hat{S}\left(t_{i}\right)^{* 1}, \hat{S}\left(t_{i}\right)^{* 2}, \cdots, \hat{S}\left(t_{i}\right)^{* B}$ para cada una de las $\boldsymbol{B}$ muestras Bootstrap.

- Estimar el error estándar del parámetro estimado $\hat{S}\left(t_{i}\right)$ calculando la desviación estándar de las $\boldsymbol{B}$ réplicas Bootstrap.

Así, obtenemos que el error estándar es 


$$
\sigma_{S\left(t_{i}\right)}^{*}=\sqrt{\frac{\sum_{b=1}^{B}\left(S\left(t_{i}\right)^{b *}-\overline{\hat{S}}\left(t_{i}\right)^{*}\right)^{2}}{(B-1)}}
$$

donde $\overline{\hat{S}}\left(t_{i}\right)^{*}$ corresponde al promedio de la estimación de la función de confiabilidad evaluada en cada tiempo $t_{i}$ de las muestras Bootstrap, y donde el procedimiento se realiza con base en los tiempos de interés $t_{i}=(0.2,0.4$ y 0.6$)$, además el procedimiento es análogo mediante el estimador no paramétrico de Nelson (1969).

\section{ESTIMADORES PARAMÉTRICOS}

Con el propósito de estudiar la eficiencia que presentan los estimadores no paramétricos frente a los estimadores paramétricos para la función de confiabilidad, debido que ante el desconocimiento de la distribución de la variable en estudio se acostumbra recurrir a los estimadores no paramétricos para la función de confiabilidad, se presentan las funciones paramétricas de los modelos generadores exponencial y Weibull.

\subsection{Función de confiabilidad modelo exponencial}

Uno de los modelos teóricos más conocidos en la práctica de análisis de confiabilidad es el exponencial, utilizado en muchos casos por facilidad de cálculo para motivos de ilustración y por su hazard constante. Supongamos que los tiempos de vida $T_{i}$ son independientes y siguen una distribución exponencial con función de densidad de probabilidad $f(t)=\lambda \exp (-\lambda t)$ y función de confiabilidad $S(t)=\exp (-\lambda t)$.

Entonces, la varianza de la función de confiabilidad en los tiempos de estudio $t_{i}=(0.2,0.4$ y 0.6$)$ es:

$$
\begin{aligned}
\operatorname{Var}(S(t))= & (\lambda t)^{2} \exp (-2 \lambda t)\left(\frac{1}{\lambda^{2}}\right) \\
& =t^{2} \exp (-2 \lambda t) \\
& =t^{2} S^{2}(t)
\end{aligned}
$$

\subsection{Función de confiabilidad modelo Weibull}

El modelo teórico Weibull presenta importantes propiedades en la práctica, ya que su capacidad de modelar diferentes comportamientos del tiempo de vida resulta de mucha utilidad. Además se utilizaron estos modelos para realizar dicha comparación de resultados con los autores referenciados. Suponemos que los tiempos de vida $T_{i}$ son independientes y siguen una distribución Weibull con función de densidad de probabilidad

$$
f(t)=\lambda \beta(\lambda t)^{\beta-1} \exp \left[-(\lambda t)^{\beta}\right]
$$

Con función de confiabilidad:

$$
S(t)=\exp \left[-(\lambda t)^{\beta}\right]
$$

Análogamente se obtiene la varianza de manera paramétrica de la función de confiabilidad en el modelo Weibull

$$
\begin{aligned}
\operatorname{Var}(S(t))= & \operatorname{Var}\left(\exp \left[-(\lambda t)^{\beta}\right]\right) \\
& =\left[\beta(\lambda t)^{\beta-1}\right]^{2}[\Gamma(1 \\
& \left.\left.+\frac{2}{\beta}\right)-\Gamma^{2}\left(1+\frac{1}{\beta}\right)\right]
\end{aligned}
$$

\section{ESTUDIO DE SIMULACIÓN}

En esta sección se presentan los resultados obtenidos al comparar estimadores paramétricos y no paramétricos para la función de confiabilidad en diferentes tiempos de interés, tomados teniendo en cuenta la literatura y con el propósito de ser comparables algunos autores referenciados que utilizaron los estimadores de Kaplan y Meier (1958) y el estimador de Nelson (1969). Además, en este trabajo se adicionan los estimadores a través del remuestreo Bootstrap aplicado a los estimadores de Kaplan y Meier y Nelson. Estos estimadores se compararon utilizando un algoritmo en R.

\section{RESULTADOS DE SIMULACIÓN}

Para realizar la comparación de los estimadores no paramétricos frente a los paramétricos, se presentan en las tablas los promedios de los errores cuadráticos medios de la simulación. Se realiza la simulación debido a la complejidad de las formas explícitas de los estimadores. Para calcular el error cuadrático medio del estimador en términos del sesgo y la varianza del mismo, se toma como referencia (parámetro) el valor 
determinado teniendo en cuenta el modelo generador de probabilidad, es decir, un valor de error cuadrático medio bajo indicará que la estimación no paramétrica se aleja de la paramétrica.

Se utilizaron 1000 muestras y 300 remuestras Bootstrap con diferentes escenarios de simulación, teniendo en cuenta las combinaciones de los modelos generadores Exponencial $(\alpha)$ y Weibull $(\alpha, \beta)$, donde $\alpha=1$ y 2 y $\beta=0.5$ para los tiempos de estudio $t_{i}=(0.2,0.4$ y 0.6$)$ con porcentajes de censura $0 \%, 25 \%$ y $50 \%$ y tamaños de muestra $\mathrm{n}=25,50$ y 100 , algunos tiempos y porcentajes de censura, teniendo en cuenta la literatura de Borgan y Liestol (1960).

Para mejor entendimiento de los resultados, se sigue la abreviatura en las tablas, KM: estimador Kaplan y Meier (1958), NA.(v.a.j): estimador Nelson (1969) con la varianza de Aalen y Johansen (1978), NA(v.h): estimador Nelson (1969) con la varianza de la hazard acumulada, Boot.KM: Bootstrap aplicado al estimador Kaplan y Meier y Boot.NA: Bootstrap aplicado al estimador Nelson.

A continuación se presentan las estimaciones paramétricas para la función de confiabilidad, considerando los modelos generadores de los tiempos observados y censurados.

Tabla 1. Estimación paramétrica de referencia

\begin{tabular}{cccc}
\hline $\begin{array}{c}\text { Modelo } \\
\text { generador }\end{array}$ & $\hat{S}(0.2)$ & $\hat{S}(0.4)$ & $\hat{S}(0.6)$ \\
\hline $\operatorname{Exp}(1)$ & 0.818731 & 0.670320 & 0.548812 \\
$\operatorname{Exp}(2)$ & 0.670320 & 0.449329 & 0.301194 \\
Weib(1,0.5) & 0.639407 & 0.531286 & 0.460890 \\
\hline
\end{tabular}

\section{Resultados $n=25$}

Hay que resaltar que los valores en negrilla en las tablas corresponden a los menores promedios de errores cuadráticos medios de cada muestra generada debido a las combinaciones de tiempos de falla (F) y censura (C), es decir, en cada fila de la tabla. En la Tabla 2 se nota que los errores cuadráticos medios del estimador KM y Boot. KM no tienen buenos resultados, con observaciones completas, mientras que en el estimador de NA con la varianza de la hazard acumulada y la alternativa de
Aalen y Johansen (1978) son mejores los resultados para los modelos generadores exponencial (E) y Weibull (W) respectivamente. Por otra parte, en la Tabla 3 tienden a mejorar los resultados para el estimador de KM.

Tabla 2. PECM con $0 \%$ de censura para $n=25$

\begin{tabular}{cccccc}
\hline \multicolumn{6}{c}{$t_{i}=0.2$} \\
\hline F/C & KM & NA.(v.a.j) & NA.(v.h) & Boot.KM & Boot.NA. \\
\hline E/E & 0.007168 & 0.006644 & $\mathbf{0 . 0 0 6 6 4 0}$ & 0.212901 & 0.212478 \\
E/W & 0.006785 & 0.006279 & $\mathbf{0 . 0 0 6 2 7 5}$ & 0.212901 & 0.212478 \\
W/E & 0.009580 & $\mathbf{0 . 0 0 9 1 2 0}$ & 0.042075 & 0.077232 & 0.076725 \\
W/W & 0.009580 & $\mathbf{0 . 0 0 9 1 2 0}$ & 0.042075 & 0.077232 & 0.076725 \\
\hline & & $t_{i}=0.4$ & & \\
\hline F/C & KM & NA.(v.a.j) & NA.(v.h) & Boot.KM & Boot.NA. \\
\hline E/E & 0.009880 & 0.009090 & $\mathbf{0 . 0 0 9 0 8 3}$ & 0.099614 & 0.099191 \\
E/W & 0.009446 & 0.008632 & $\mathbf{0 . 0 0 8 6 2 4}$ & 0.099614 & 0.099191 \\
W/E & 0.024688 & $\mathbf{0 . 0 2 1 6 4 6}$ & 0.070619 & 0.031301 & 0.030794 \\
W/W & 0.024688 & $\mathbf{0 . 0 2 1 6 4 6}$ & 0.070619 & 0.031301 & 0.030794 \\
\hline & & \multicolumn{5}{c}{$t_{i}=0.6$} & & \\
\hline F/C & KM & NA.(v.a.j) & NA.(v.h) & Boot.KM & Boot.NA. \\
E/E & 0.010800 & 0.009928 & $\mathbf{0 . 0 0 9 9 1 8}$ & 0.099660 & 0.039237 \\
E/W & 0.010622 & 0.009870 & $\mathbf{0 . 0 0 9 8 6 0}$ & 0.099660 & 0.039237 \\
W/E & 0.046729 & 0.040873 & 0.080366 & 0.013964 & $\mathbf{0 . 0 1 3 4 5 7}$ \\
W/W & 0.046729 & 0.040873 & 0.080366 & 0.013964 & $\mathbf{0 . 0 1 3 4 5 7}$ \\
\hline
\end{tabular}

Tabla 3. PECM con $25 \%$ de censura para $n=25$

\begin{tabular}{cccccc}
\hline \multicolumn{6}{c}{$t_{i}=0.2$} \\
\hline F/C & KM & NA.(v.a.j) & NA.(v.h) & Boot.KM & Boot.NA. \\
\hline E/E & 0.005357 & 0.005241 & $\mathbf{0 . 0 1 2 8 9 2}$ & 0.144075 & 0.143309 \\
E/W & 0.004847 & 0.004776 & $\mathbf{0 . 0 0 4 7 7 3}$ & 0.144198 & 0.143437 \\
W/E & $\mathbf{0 . 0 1 2 3 9 3}$ & 0.012919 & 0.018541 & 0.049119 & 0.048592 \\
W/W & $\mathbf{0 . 0 1 2 4 2 5}$ & 0.012892 & 0.020084 & 0.042390 & 0.048592 \\
\hline \multicolumn{5}{c}{$t_{i}=0.4$} \\
\hline F/C & KM & NA.(v.a.j) & NA.(v.h) & Boot.KM & Boot.NA. \\
\hline E/E & $\mathbf{0 . 0 0 9 4 6 3}$ & 0.009523 & 0.009515 & 0.055994 & 0.055228 \\
E/W & $\mathbf{0 . 0 0 7 7 6 5}$ & 0.007873 & 0.007865 & 0.056053 & 0.055293 \\
W/E & 0.009138 & $\mathbf{0 . 0 0 8 7 4 1}$ & 0.026381 & 0.015239 & 0.014713 \\
W/W & 0.010940 & $\mathbf{0 . 0 1 0 0 3 4}$ & 0.032472 & 0.012753 & 0.012054 \\
\hline \multicolumn{7}{c}{$t_{i}=0.6$} \\
\hline F/C & KM & NA.(v.a.j) & NA.(v.h) & Boot.KM & Boot.NA. \\
E/E & $\mathbf{0 . 0 1 2 2 2 4}$ & 0.012439 & 0.012425 & 0.016678 & 0.015912
\end{tabular}




\begin{tabular}{rccccc}
$\mathrm{E} / \mathrm{W}$ & $\mathbf{0 . 0 1 3 0 8 8}$ & 0.013382 & 0.013367 & 0.016684 & 0.015924 \\
$\mathrm{~W} / \mathrm{E}$ & 0.012242 & 0.010289 & 0.026631 & 0.005747 & $\mathbf{0 . 0 0 5 2 2 1}$ \\
$\mathrm{W} / \mathrm{W}$ & 0.02020 & 0.016684 & 0.038821 & 0.006023 & $\mathbf{0 . 0 0 5 3 2 5}$ \\
\hline
\end{tabular}

En la Tabla 4 se nota que cuando se aumenta el porcentaje de censura en tamaños de muestra pequeños, el cual sería en situaciones extremas, se observa que el $50 \%$ de los mejores resultados se presenta con Boot.NA.

Tabla 4. $P E C M$ con $50 \%$ de censura para $n=25$

\begin{tabular}{cccccc}
\hline \multicolumn{6}{c}{$t_{i}=0.2$} \\
\hline F/C & KM & NA.(v.a.j) & NA.(v.h) & Boot.KM & Boot.NA. \\
\hline E/E & $\mathbf{0 . 0 0 9 0 9 1}$ & 0.009270 & 0.009268 & 0.062696 & 0.061533 \\
E/W & $\mathbf{0 . 0 0 8 3 9 0}$ & 0.008587 & 0.008585 & 0.062322 & 0.061149 \\
W/E & 0.033085 & 0.034242 & $\mathbf{0 . 0 0 5 1 1 8}$ & 0.008593 & 0.008279 \\
W/W & 0.031830 & 0.033003 & $\mathbf{0 . 0 0 6 1 9 9}$ & 0.008015 & 0.007282 \\
\hline & \multicolumn{5}{c}{$t_{i}=0.4$} \\
\hline F/C & KM & NA.(v.a.j) & NA.(v.h) & Boot.KM & Boot.NA. \\
\hline E/E & 0.021534 & 0.022431 & 0.022423 & 0.014204 & $\mathbf{0 . 0 1 3 0 4 0}$ \\
E/W & 0.020588 & 0.021504 & 0.021496 & 0.014041 & $\mathbf{0 . 0 1 2 8 6 8}$ \\
W/E & 0.029720 & 0.031615 & 0.006776 & 0.002819 & $\mathbf{0 . 0 0 2 5 0 6}$ \\
W/W & 0.024052 & 0.025769 & 0.008431 & 0.006212 & $\mathbf{0 . 0 0 5 4 7 9}$ \\
\hline & \multicolumn{5}{c}{$t_{i}=0.6$} \\
F/C & KM & NA.(v.a.j) & NA.(v.h) & Boot.KM & Boot.NA. \\
E/E & 0.030435 & 0.032228 & 0.032207 & 0.007299 & $\mathbf{0 . 0 0 6 1 3 5}$ \\
E/W & 0.029723 & 0.031490 & 0.031468 & 0.007310 & $\mathbf{0 . 0 0 6 1 3 7}$ \\
W/E & 0.023414 & 0.025648 & $\mathbf{0 . 0 0 9 0 8 2}$ & 0.011627 & 0.011313 \\
W/W & 0.015965 & 0.016931 & $\mathbf{0 . 0 1 0 5 8 4}$ & 0.017605 & 0.016873 \\
\hline & & & & &
\end{tabular}

Resultados $n=50$

Tabla 5. PECM con $0 \%$ de censura para $n=50$

\begin{tabular}{cccccc}
\hline \multicolumn{6}{c}{$t_{i}=0.2$} \\
\hline F/C & KM & NA.(v.a.j) & NA.(v.h) & Boot.KM & Boot.NA. \\
\hline E/E & 0.003458 & 0.003325 & $\mathbf{0 . 0 0 3 3 2 4}$ & 0.205184 & 0.204979 \\
E/W & 0.003242 & 0.003116 & $\mathbf{0 . 0 0 3 1 1 5}$ & 0.205184 & 0.204979 \\
W/E & $\mathbf{0 . 0 0 4 7 7 6}$ & 0.004782 & 0.031217 & 0.072325 & 0.072090 \\
W/W & $\mathbf{0 . 0 0 4 7 7 6}$ & 0.004782 & 0.031217 & 0.072325 & 0.072090 \\
\hline & & & $t_{i}=0.4$ \\
F/C & KM & NA.(v.a.j) & NA.(v.h) & Boot.KM & Boot.NA. \\
\hline E/E & 0.004749 & 0.004596 & $\mathbf{0 . 0 0 4 5 9 5}$ & 0.094471 & 0.094265 \\
E/W & 0.003772 & 0.003585 & $\mathbf{0 . 0 0 3 5 8 4}$ & 0.094471 & 0.094265 \\
W/E & 0.015267 & $\mathbf{0 . 0 1 4 0 6 6}$ & 0.059486 & 0.028536 & 0.028302
\end{tabular}

\begin{tabular}{cccccc} 
W/W & 0.015267 & $\mathbf{0 . 0 1 4 0 6 6}$ & 0.059486 & 0.028536 & 0.028302 \\
\hline \multicolumn{5}{c}{$t_{i}=0.6$} \\
\hline F/C & KM & NA.(v.a.j) & NA.(v.h) & Boot.KM & Boot.NA. \\
E/E & 0.005294 & 0.005044 & $\mathbf{0 . 0 0 5 0 4 3}$ & 0.036624 & 0.036418 \\
E/W & 0.004928 & 0.004720 & $\mathbf{0 . 0 0 4 7 1 9}$ & 0.036624 & 0.036418 \\
W/E & 0.035562 & 0.033004 & 0.070509 & 0.012594 & $\mathbf{0 . 0 1 2 3 5 9}$ \\
W/W & 0.035562 & 0.033004 & 0.070509 & 0.012594 & $\mathbf{0 . 0 1 2 3 5 9}$ \\
\hline
\end{tabular}

Aumentando el tamaño de muestra con las observaciones completas, se nota que el estimador NA con la varianza tradicional tiene mejores resultados para los tiempos exponenciales.

Tabla 6. $\mathrm{PECM}$ con $25 \%$ de censura para $\mathrm{n}=50$

\begin{tabular}{cccccc}
\hline \multicolumn{6}{c}{$t_{i}=0.2$} \\
\hline F/C & KM & NA.(v.a.j) & NA.(v.h) & Boot.KM & Boot.NA. \\
\hline E/E & 0.003256 & 0.003280 & $\mathbf{0 . 0 0 3 2 8 0}$ & 0.137738 & 0.137338 \\
E/W & 0.003146 & 0.003181 & $\mathbf{0 . 0 0 3 1 8 0}$ & 0.137782 & 0.137372 \\
W/E & $\mathbf{0 . 0 1 1 2 8 9}$ & 0.011707 & 0.011681 & 0.046183 & 0.045913 \\
W/W & $\mathbf{0 . 0 1 0 6 8 9}$ & 0.011093 & 0.012758 & 0.039516 & 0.039166 \\
\hline & \multicolumn{5}{c}{$t_{i}=0.4$} \\
\hline F/C & KM & NA.(v.a.j) & NA.(v.h) & Boot.KM & Boot.NA. \\
\hline E/E & $\mathbf{0 . 0 0 5 9 9 5}$ & 0.006175 & 0.006174 & 0.052201 & 0.051801 \\
E/W & $\mathbf{0 . 0 0 5 0 3 7}$ & 0.005237 & 0.005236 & 0.052213 & 0.051803 \\
W/E & $\mathbf{0 . 0 0 4 7 3 3}$ & 0.004783 & 0.019044 & 0.013924 & 0.013654 \\
W/W & 0.005250 & $\mathbf{0 . 0 0 5 1 0 0}$ & 0.024176 & 0.011625 & 0.011275 \\
\hline & \multicolumn{5}{c}{$t_{i}=0.6$} \\
F/C & KM & NA.(v.a.j) & NA.(v.h) & Boot.KM & Boot.NA. \\
E/E & $\mathbf{0 . 0 0 7 5 9 5}$ & 0.007935 & 0.007933 & 0.014967 & 0.014667 \\
E/W & $\mathbf{0 . 0 0 7 6 9 6}$ & 0.008062 & 0.008060 & 0.014951 & 0.014542 \\
W/E & 0.006602 & $\mathbf{0 . 0 0 5 9 1 6}$ & 0.020784 & 0.005488 & 0.005217 \\
W/W & 0.011216 & $\mathbf{0 . 0 0 9 9 5 5}$ & 0.030103 & 0.006033 & 0.005682 \\
\hline & & & &
\end{tabular}

Tabla 7. PECM con $50 \%$ de censura para $\mathrm{n}=50$

\begin{tabular}{cccccc}
\hline \multicolumn{6}{c}{$t_{i}=0.2$} \\
\hline F/C & KM & NA.(va.j) & NA.(v.h) & Boot.KM & Boot.NA. \\
\hline E/E & $\mathbf{0 . 0 0 7 4 0 6}$ & 0.007540 & 0.007540 & 0.064637 & 0.064039 \\
E/W & $\mathbf{0 . 0 0 7 2 5 0}$ & 0.007387 & 0.007387 & 0.064542 & 0.063946 \\
W/E & 0.032574 & 0.033205 & $\mathbf{0 . 0 0 2 7 1 0}$ & 0.010081 & 0.009897 \\
W/W & 0.030534 & 0.031187 & $\mathbf{0 . 0 0 3 1 1 8 7}$ & 0.009062 & 0.008660 \\
\hline \multicolumn{6}{c}{$t_{i}=0.4$} \\
\hline F/C & KM & NA.(va.j) & NA.(v.h) & Boot.KM & Boot.NA. \\
\hline E/E & 0.018758 & 0.019303 & 0.019302 & 0.014962 & $\mathbf{0 . 0 1 4 3 6 4}$
\end{tabular}




\begin{tabular}{cccccc} 
E/W & 0.017798 & 0.018349 & 0.018348 & 0.014918 & $\mathbf{0 . 0 1 4 3 2 1}$ \\
W/E & 0.026658 & 0.027670 & 0.003665 & 0.002512 & $\mathbf{0 . 0 0 2 3 2 7}$ \\
W/W & 0.020257 & 0.021221 & $\mathbf{0 . 0 0 4 5 7 8}$ & 0.005706 & 0.005304 \\
\hline \multicolumn{5}{c}{$t_{i}=0.6$} \\
\hline F/C & KM & NA.(v.a.j) & NA.(v.h) & Boot.KM & Boot.NA. \\
E/E & 0.027008 & 0.028105 & 0.028103 & 0.007089 & $\mathbf{0 . 0 0 6 4 9 2}$ \\
E/W & 0.029844 & 0.030965 & 0.030963 & 0.007086 & $\mathbf{0 . 0 0 6 4 9 0}$ \\
W/E & 0.018937 & 0.020095 & $\mathbf{0 . 0 0 5 1 5 5}$ & 0.010150 & 0.009966 \\
W/W & 0.009975 & 0.010738 & $\mathbf{0 . 0 0 5 1 4 1}$ & 0.016087 & 0.015686 \\
\hline
\end{tabular}

Resultados $n=100$

Tabla 8. PECM con $0 \%$ de censura para $\mathrm{n}=100$

\begin{tabular}{cccccc}
\hline \multicolumn{5}{c}{$t_{i}=0.2$} \\
\hline F/C & KM & NA.(va.j) & NA.(v.h) & Boot.KM & Boot.NA. \\
\hline E/E & 0.001581 & 0.001548 & $\mathbf{0 . 0 0 1 5 4 8}$ & 0.199682 & 0.199572 \\
E/W & 0.001502 & 0.001477 & $\mathbf{0 . 0 0 1 4 7 7}$ & 0.199682 & 0.199572 \\
W/E & $\mathbf{0 . 0 0 2 8 9 6}$ & 0.002945 & 0.026952 & 0.069610 & 0.069462 \\
W/W & $\mathbf{0 . 0 0 2 8 9 6}$ & 0.002945 & 0.026952 & 0.069610 & 0.069462 \\
\hline & & & $t_{i}=0.4$ & & \\
\hline F/C & KM & NA.(v.a.j) & NA.(v.h) & Boot.KM & Boot.NA. \\
\hline E/E & 0.002294 & $\mathbf{0 . 0 0 2 2 4 7}$ & 0.002247 & 0.090808 & 0.090698 \\
E/W & 0.002019 & $\mathbf{0 . 0 0 1 9 9 0}$ & 0.001990 & 0.090808 & 0.090698 \\
W/E & 0.010973 & $\mathbf{0 . 0 1 0 4 4 2}$ & 0.054512 & 0.026987 & 0.026838 \\
W/W & 0.010973 & $\mathbf{0 . 0 1 0 4 4 2}$ & 0.054512 & 0.026987 & 0.026838 \\
\hline & & & $t_{i}=0.6$ & & \\
\hline F/C & KM & NA.(v.a.j) & NA.(v.h) & Boot.KM & Boot.NA. \\
E/E & 0.002396 & $\mathbf{0 . 0 0 2 3 4 0}$ & 0.002340 & 0.034467 & 0.034357 \\
E/W & 0.002261 & $\mathbf{0 . 0 0 2 2 2 2}$ & 0.002222 & 0.034467 & 0.034357 \\
W/E & 0.030479 & 0.029280 & 0.066010 & 0.011803 & $\mathbf{0 . 0 1 1 6 5 4}$ \\
W/W & 0.030479 & 0.029280 & 0.066010 & 0.011803 & $\mathbf{0 . 0 1 1 6 5 4}$ \\
\hline & & & & &
\end{tabular}

Tabla 9. $\mathrm{PECM}$ con $25 \%$ de censura para $\mathrm{n}=100$

\begin{tabular}{cccccc}
\hline \multicolumn{6}{c}{$t_{i}=0.2$} \\
\hline F/C & KM & NA.(v.a.j) & NA.(v.h) & Boot.KM & Boot.NA. \\
\hline E/E & 0.002299 & 0.002336 & $\mathbf{0 . 0 0 2 3 3 6}$ & 0.130407 & 0.130183 \\
E/W & 0.002341 & 0.002381 & $\mathbf{0 . 0 0 2 3 8 1}$ & 0.130347 & 0.130127 \\
W/E & 0.011667 & 0.011913 & $\mathbf{0 . 0 0 8 2 2 2}$ & 0.042990 & 0.042819 \\
W/W & 0.010734 & 0.010977 & $\mathbf{0 . 0 0 9 2 0 6}$ & 0.036461 & 0.036256 \\
\hline & \multicolumn{7}{c}{$t_{i}=0.4$} \\
\hline F/C & KM & NA.(v.a.j) & NA.(v.h) & Boot.KM & Boot.NA. \\
\hline E/E & 0.005004 & $\mathbf{0 . 0 0 5 1 4 7}$ & 0.005147 & 0.047860 & 0.047636 \\
E/W & 0.005355 & $\mathbf{0 . 0 0 5 5 1 4}$ & 0.005514 & 0.047833 & 0.047612
\end{tabular}

\begin{tabular}{cccccc} 
W/E & $\mathbf{0 . 0 0 2 8 6 1}$ & 0.002966 & 0.014350 & 0.012377 & 0.012206 \\
W/W & $\mathbf{0 . 0 0 2 6 1 6}$ & 0.002630 & 0.019119 & 0.010358 & 0.010153 \\
\hline \multicolumn{6}{c}{$t_{i}=0.6$} \\
\hline F/C & KM & NA.(v.a.j) & NA.(v.h) & Boot.KM & Boot.NA. \\
E/E & 0.006268 & $\mathbf{0 . 0 0 6 5 1 9}$ & 0.006519 & 0.013073 & 0.012849 \\
E/W & 0.006713 & $\mathbf{0 . 0 0 6 9 8 0}$ & 0.006980 & 0.013073 & 0.012852 \\
W/E & 0.003000 & $\mathbf{0 . 0 0 2 7 8 7}$ & 0.015476 & 0.005012 & 0.004841 \\
W/W & 0.006541 & $\mathbf{0 . 0 0 6 0 5 4}$ & 0.024390 & 0.005930 & 0.005725 \\
\hline
\end{tabular}

Cuando aumenta el tamaño de muestra a $\mathrm{n}=100$, se mantienen en general los resultados, sin embargo, vale resaltar que el estimador de Kaplan y Meier (1958) que

Tabla 10. PECM con $50 \%$ de censura para $\mathrm{n}=100$

\begin{tabular}{cccccc}
\hline \multicolumn{6}{c}{$t_{i}=0.2$} \\
\hline F/C & KM & NA.(v.a.j) & NA.(v.h) & Boot.KM & Boot.NA. \\
\hline E/E & $\mathbf{0 . 0 0 6 9 5 3}$ & 0.007032 & 0.007032 & 0.061784 & 0.061403 \\
E/W & $\mathbf{0 . 0 0 6 9 2 8}$ & 0.007008 & 0.007008 & 0.061690 & 0.061315 \\
W/E & 0.034219 & 0.034548 & $\mathbf{0 . 0 0 1 2 9 8}$ & 0.009261 & 0.009171 \\
W/W & 0.031798 & 0.032144 & $\mathbf{0 . 0 0 1 4 8 2}$ & 0.008505 & 0.008262 \\
\hline & \multicolumn{5}{c}{$t_{i}=0.4$} \\
\hline F/C & KM & NA.(v.a.j) & NA.(v.h) & Boot.KM & Boot.NA. \\
\hline E/E & 0.018079 & 0.018375 & 0.018375 & 0.013696 & $\mathbf{0 . 0 1 3 3 1 4}$ \\
E/W & 0.018403 & 0.018704 & 0.018704 & 0.013681 & $\mathbf{0 . 0 1 3 3 0 5}$ \\
W/E & 0.027328 & 0.02864 & $\mathbf{0 . 0 0 2 2 3 9}$ & 0.002598 & 0.002507 \\
W/W & 0.019713 & 0.020243 & $\mathbf{0 . 0 0 2 2 7 2}$ & 0.005925 & 0.005682 \\
\hline & \multicolumn{5}{c}{$t_{i}=0.6$} \\
F/C & KM & NA.(v.a.j) & NA.(v.h) & Boot.KM & Boot.NA. \\
E/E & 0.025425 & 0.026014 & 0.026014 & 0.007121 & $\mathbf{0 . 0 0 6 7 4 0}$ \\
E/W & 0.026746 & 0.027346 & 0.027346 & 0.007172 & $\mathbf{0 . 0 0 6 7 9 6}$ \\
W/E & 0.019366 & 0.019992 & $\mathbf{0 . 0 0 3 9 8 3}$ & 0.010826 & 0.010736 \\
W/W & 0.008893 & 0.009373 & $\mathbf{0 . 0 0 2 5 9 5}$ & 0.016812 & 0.016569 \\
\hline
\end{tabular}

se utiliza tradicionalmente, no siempre tiene mejores resultados, ya que se puede notar que la estimación mediante el remuestreo al estimador de Kaplan y Meier tiene mejores resultados, particularmente cuando se tienen datos de modelo generador Weibull. Por otra parte, cuando se tienen escenarios extremos de alto porcentaje de censura, se afecta considerablemente la estimación de la función de confiabilidad en los tiempos de interés estudiados, además muestra lo mencionado por Borgan y Liestol (1990) en el sentido de que el estimador de Kaplan y Meier presenta mejores resultados cuando 
tenemos modelos generadores exponencial y tiempos $\mathrm{t}=0.2$ y 0.6 .

\section{APLICACIÓN}

Para la aplicación se toman los datos de Nelson (1982, p. 133), que corresponden a los tiempos de falla de 70 ventiladores. Aplicando una prueba Kolmogorov-Smirnov se tiene un Pvalor $<0.05$ que indica que los tiempos se ajustan significativamente a una Weibull $(\lambda=1.8, \beta=5540.7)$. Así, tomando como tiempo de interés el primer cuartil $t_{0.25}=2210$ se tiene que la función de confiabilidad paramétrica calculada $\hat{S}(2110)=\exp -(2110 * 1.8)^{1 / 5540.7} \approx 0.4$, teniendo en cuenta que el porcentaje de censura es muy

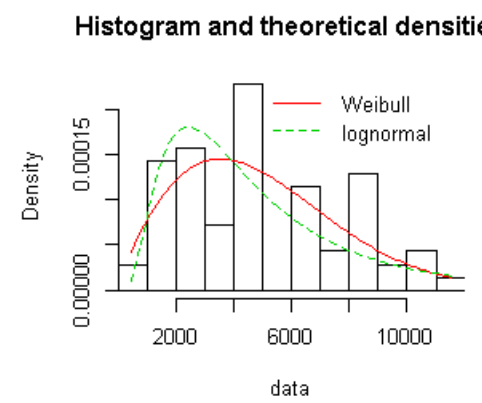

Empirical and theoretical CDFs

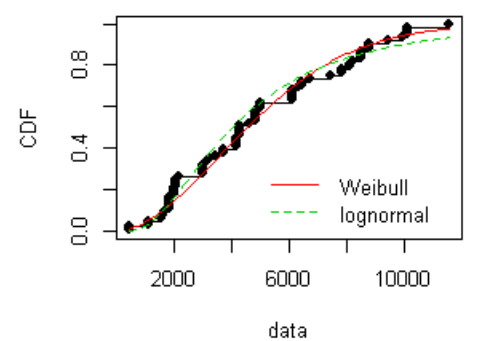

extremo del $83 \%$, entonces $\hat{S}_{K M}(2110)=0.890622$. $\hat{S}_{N A}(2110)=0.89198, \quad \hat{S}_{\text {Boot.KM }}(2110)=0.98571$, $\hat{S}_{\text {Boot.NA }}(2110)=0.98582$, con errores cuadráticos medios respectivamente $\mathrm{ECM}_{\mathrm{KM}}=0.79475$, $\mathrm{ECM}_{\mathrm{NA}}=0.79713, \quad \mathrm{ECM}_{\text {Boot.KM }}=0.97197$, $\mathrm{ECM}_{\text {Boot.NA }}=0.97227$. Vale resaltar que el remuestreo Bootstrap mejora los resultados en situaciones extremas de censura, además los estimadores tradicionales KM y NA resultan muy similares, siendo mejor el ECM de KM. En general, los estimadores utilizados en situaciones extremas se manifiestan muy alejados de la estimación paramétrica; razón por la cual se recomienda tener cuidado en el uso de estos estimadores.

Gráficamente el ajuste de los datos es:
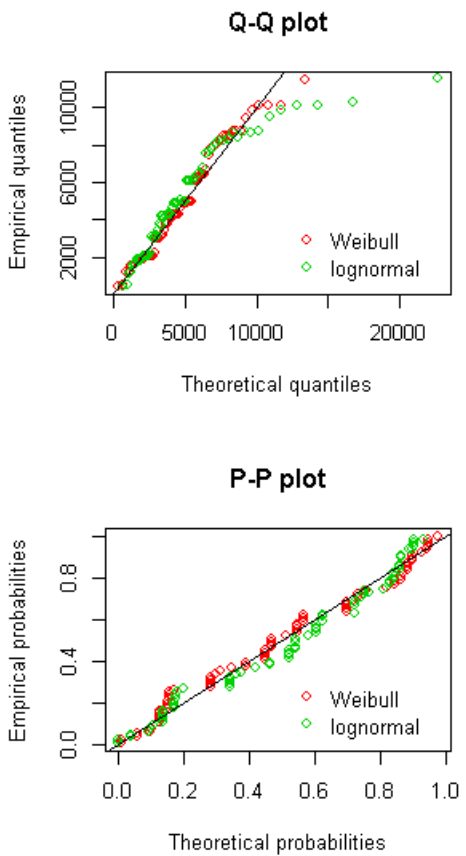

Figura 1. Ajuste de datos
Se nota el buen ajuste de los datos al modelo Weibull, confirmado con la prueba respectiva.

\section{CONCLUSIONES}

- Se verifica que los estimadores no paramétricos tienden a ser más eficientes a medida que el tamaño de muestra aumenta; además, con porcentajes de censura bajos, coinciden con los resultados de
Ramírez (2011) en los intervalos de confianza, para el tiempo $t=0.2$ y Borgan y Liestol (1990) para los demás tiempos.

El estimador de Nelson (1969) tiene mejores resultados que el estimador de Kaplan y Meier (1958), cuando los tamaños muestrales son más pequeños y en particular para modelos generadores exponencial. 
El método de remuestreo Bootstrap al estimador de Nelson (1969), presenta mejores resultados que el estimador de Kaplan y Meier (1958), atendiendo los escenarios planteados.

\section{REFERENCIAS}

Aalen O. \& Johansen S. (1978). An Empirical Transition Matrix for Nonhomogeneus Markov Chains Based on Censored Observations. Scandinavian Journal of Statistics, 5, 141-150.

Akritas M. (1986). Bootstrapping the Kaplan-Meier Estimator.AmericanStatisticalAssociation, 81,1032 1138. doi: 10.1080/01621459.1986.10478369

Arrabal, C., Dos Santos, K., Da Rocha, R. Nonaka, R. \& Meira, S. (2014). Comparison of resampling method applied to censored data. International Journal of Advanced Statistics and Probability, 2(2), 48-55. doi: 10.14419/ijasp.v2i2.2291

Borgan, Ø.\& Liestol, K. (1990). A Note on Confidence Intervals and Bands for the Survival Function Based on Transformations. Scandinavian Journal of Statistics, 17(1), 35-41.

Colosimo, E. \& Giolo, S. (2006). Análise de sobrevivencia aplicada, En E. Blucher (ed.). Belem: Universidade Federale do Pará.

Greenwood, M. (1926). The natural duration of cancer. Reports on Public Health and Medical Subjects, 33, 1-26. Record Number 19272700028
He, P., Kong, G. \& Su, Z. (2013). Estimating the survival functions for right-censored and interval-censored data with piecewise constant hazard functions. Contemporary Clinical Trials, 35, 122-127. doi: http://dx.doi.org/10.1016/j. cet.2013.04.009

Kalbfleisch, J. \& Prentice, R. (1980). The statistical analysis of failure time data. New York: Wiley. doi: 10.1002/9781118032985

Kaplan, E. \& Meier, P. (1958). Estimation from Incomplete Observations. American Statistical Association, 53, 457-481. doi: 10.1080/01621459.1 958.10501452

Lawless, J. (2003). Statistical Models and Methods for Lifetime Data. New York: Wiley and Sons. doi: 10.1002/9781118033005.fmatter

Nelson, W. (1969). Hazard plotting for incomplete failure data. Journal of Quality Technology, 61, 27-52.

Nelson, W. (1982). Applied Life Data Analysis, New York: John Wiley Sons. doi: 10.1002/ bimj.4710270615

Ramírez, J. (2011). Comparación de intervalos de confianza para la función de supervivencia con censura a derecha. Revista Colombiana de Estadistica, 34, 197-209. 